Louisiana State University

LSU Digital Commons

\title{
The dust budget of the small magellanic cloud: Are asymptotic giant branch stars the primary dust source at low metallicity?
}

\author{
M. L. Boyer \\ Space Telescope Science Institute \\ S. Srinivasan \\ Institut d'Astrophysique de Paris \\ D. Riebel \\ Johns Hopkins University \\ I. McDonald \\ The University of Manchester \\ J. Th Van Loon \\ Keele University
}

See next page for additional authors

Follow this and additional works at: https://digitalcommons.Isu.edu/physics_astronomy_pubs

\section{Recommended Citation}

Boyer, M., Srinivasan, S., Riebel, D., McDonald, I., Van Loon, J., Clayton, G., Gordon, K., Meixner, M., Sargent, B., \& Sloan, G. (2012). The dust budget of the small magellanic cloud: Are asymptotic giant branch stars the primary dust source at low metallicity?. Astrophysical Journal, 748 (1) https://doi.org/10.1088/ 0004-637X/748/1/40 


\section{Authors}

M. L. Boyer, S. Srinivasan, D. Riebel, I. McDonald, J. Th Van Loon, G. C. Clayton, K. D. Gordon, M. Meixner, B. A. Sargent, and G. C. Sloan 
Draft VERSion OCTOBER 29, 2018

Preprint typeset using $\mathrm{L}^{A} \mathrm{~T}_{\mathrm{E}} \mathrm{X}$ style emulateapj v. 04/20/08

\title{
THE DUST BUDGET OF THE SMC: ARE AGB STARS THE PRIMARY DUST SOURCE AT LOW METALLICITY?
}

\author{
M. L. Boyer ${ }^{1}$, S. Srinivasan ${ }^{2}$, D. Riebel ${ }^{3}$, I. McDonald $^{4}$, J. Th. van Loon ${ }^{5}$, G. C. Clayton ${ }^{6}$, K. D. Gordon $^{1}$, \\ M. Meixner ${ }^{1}$, B. A. Sargent ${ }^{1}$, G. C. Sloan $^{7}$ \\ (Received; Revised; Accepted) \\ Draft version October 29, 2018
}

\begin{abstract}
We estimate the total dust input from the cool evolved stars in the Small Magellanic Cloud (SMC), using the 8- $\mu \mathrm{m}$ excess emission as a proxy for the dust-production rate. We find that Asymptotic Giant Branch (AGB) and red supergiant (RSG) stars produce $(8.6-9.5) \times 10^{-7} M_{\odot} \mathrm{yr}^{-1}$ of dust, depending on the fraction of far-infrared sources that belong to the evolved star population (with 10\%-50\% uncertainty in individual dust-production rates). RSGs contribute the least $(<4 \%)$, while carbon-rich AGB stars (especially the so-called "extreme" AGB stars) account for $87 \%-89 \%$ of the total dust input from cool evolved stars. We also estimate the dust input from hot stars and supernovae ( $\mathrm{SNe})$, and find that if SNe produce $10^{-3} M_{\odot}$ of dust each, then the total SN dust input and AGB input are roughly equivalent. We consider several scenarios of SNe dust production and destruction and find that the interstellar medium (ISM) dust can be accounted for solely by stellar sources if all SNe produce dust in the quantities seen around the dustiest examples and if most SNe explode in dense regions where much of the ISM dust is shielded from the shocks. We find that AGB stars contribute only $2.1 \%$ of the ISM dust. Without a net positive contribution from SNe to the dust budget, this suggests that dust must grow in the ISM or be formed by another unknown mechanism.
\end{abstract}

Subject headings: stars: AGB - ISM: dust, extinction-galaxies: Magellanic Clouds - stars: supernovae

\section{INTRODUCTION}

Dust in galaxies plays an important role in allowing molecular clouds to cool sufficiently to form stars and planets. Dust is known to form in the atmospheres of stars or in explosive/eruptive events such as novae and supernovae (SNe). There is no known mechanism for forming dust in the interstellar medium (ISM), though it may be possible to grow dust in the ISM from existing dust grains (e.g., Dwek 1998; Draine 2009). The rate of grain growth within the ISM can be estimated by knowing the dust-injection rate from stellar sources and the dust lifetime within a particular galaxy.

Asymptotic Giant Branch (AGB) stars are important dust creators in galaxies. Recent work, however, has questioned the long-held belief (Gehrz 1989) that they are the primary dust factories. For the case of $\mathrm{J} 114816+525150$, one of the most distant quasars $(z=$ 6.4), Valiante et al. (2009) argue that AGB stars must be the source of the observed dust $\left(2 \times 10^{8} M_{\odot}\right)$, but Dwek \& Cherchneff (2011) argue that SNe can produce the observed dust if the star formation history is assumed

\footnotetext{
${ }^{1}$ STScI, 3700 San Martin Drive, Baltimore, MD 21218 USA; mboyer@stsci.edu

2 UPMC-CNRS UMR7095, Institut d'Astrophysique de Paris, F-75014 Paris, France

${ }^{3}$ Department of Physics and Astronomy, The Johns Hopkins University, Homewood Campus, Baltimore, MD 21218 USA

${ }^{4}$ Jodrell Bank Centre for Astrophysics, Alan Turing Building, University of Manchester, M13 9PL, UK

${ }^{5}$ Astrophysics Group, Lennard-Jones Laboratories, Keele University, Staffordshire ST5 5BG, UK

${ }^{6}$ Louisiana State University, Department of Physics \& Astronomy, 233-A Nicholson Hall, Tower Dr., Baton Rouge, LA 708034001, USA

7 Astronomy Department, Cornell University, Ithaca, NY 148536801, USA
}

to have long periods of very low star formation rates. SNe are generally thought incapable of producing enough dust to account for the amount seen in the ISM $\left(\lesssim 0.01 M_{\odot}\right.$ each; Andrews et al. 2011, and references therein). Surprisingly, Matsuura et al. (2011) recently detected nearly $1 M_{\odot}$ of dust around SN1987A (see also Lakicevic et al. 2011); the equivalent dust-injection rate requires $10^{7}$ $10^{10}$ dusty AGB stars. However, it is unknown whether SNe have a net positive or negative impact on dust production, as they are also efficient dust destroyers.

The only way to compare the total dust input from the evolved stars in a galaxy to the total dust budget is to detect the entire population of dusty stars at infrared (IR) wavelengths and estimate the dust-injection rate of each. These sorts of global measurements are difficult in our Galaxy owing to obscuration by the Galactic Plane and in Local Group dwarf galaxies owing to limited sensitivity and resolution (cf. Bover et al. 2009b). Only for the Magellanic Clouds has the appropriate wavelength coverage, sensitivity, spatial coverage, and resolution been achieved to attempt to derive the total dust budget of a star-forming galaxy. In the Large Magellanic Cloud (LMC), Matsuura et al. (2009) find that AGB stars and SNe combined account for only $3 \%$ of the ISM dust, though this could be much higher if other SNe remnants have large dust reservoirs like that around SN 1987A.

Here, we estimate the current global dust production from the entire population of AGB and red supergiant (RSG) stars in the Small Magellanic Cloud (SMC). With a metallicity that is $2.5-3$ times lower than the LMC (e.g., Russell \& Dopita 1992; Luck et al. 1998), the SMC is more representative of high-redshift galaxies. Combined with estimates of dust production in SNe, we estimate the total dust budget of the SMC and compare the results to measurements of the ISM dust mass. 


\subsection{Estimating Dust-Production Rates in Evolved Stars}

The effect of metallicity on the amount and type of dust species produced by an evolved star is not yet well understood. Previous studies (e.g., Groenewegen et al. 2007; Matsuura et al. 2007; Sloan et al. 2008) find that more metal-rich stars do appear to produce more oxygenrich dust. Presumably, this is because O-rich dust production is limited by the metallicity-dependent availability of oxygen and silicon, while C-rich dust production is limited by the mostly metallicity-independent production of carbon by the star itself. Metal-poor stars are thus more likely to produce C-rich dust, as any oxygen is quickly tied up in CO molecules after dredge-up, leaving an excess of carbon. However, there is evidence that even carbon stars produce less dust at low metallicity (e.g., van Loon 2000; van Loon et al. 2008), possibly due to a lack of nucleation seeds.

To accurately estimate the total amount of dust production around a star, detailed radiative transfer modeling of a well-sampled spectral energy distribution (SED) is necessary. In addition, the inclusion of an IR spectrum to determine the dust species is ideal. Studies using this method have been carried out for subsets of the dust-producing population in several galaxies and clusters spanning a wide range of metallicity (e.g., van Loon et al. 2005; Matsuura et al. 2007; Groenewegen et al. 2007; Bover et al. 2009a; Groenewegen et al. 2009a,b; Lagadec et al. 2009; McDonald et al. 2009, 2011a). However, it is currently unfeasible to use this method to measure the global dust input of an entire population of dust-producing stars since detailed radiative transfer modeling of thousands of individual stars is a prohibitively long process. One must thus depend on photometric techniques for estimating the dust input, usually in the form of an IR color analysis since IR colors generally scale with the mass-loss rate (e.g., Groenewegen 2006; Sloan et al. 2008; Matsuura et al. 2009). In this work, a full SED from optical to IR wavelengths is available for each SMC evolved star, allowing us to use the more physical approach of computing the IR excess over the stellar photosphere as a proxy for the dust production (cf. Srinivasan et al. 2009) rather than relying on the IR colors alone.

\section{DATA \& ANALYSIS}

The photometric data used in this study are from the Spitzer Legacy program "Surveying the Agents of Galaxy Evolution in the SMC", or SAGE-SMC. Images were obtained at 3.6, 4.5, 5.8, 8.0, 24, 70, and $160 \mu \mathrm{m}$ covering 30 $\mathrm{deg}^{2}$, including the Tail, Wing, and Bar of the SMC. Optical to near-IR photometry from the Magellanic Clouds Photometric Survey (MCPS; Zaritskv et al. 2002), InfraRed Survey Facility (IRSF; Kato et al.|2007), and the 2-Micron All Sky Survey (2MASS; Skrutskie et al.|2006) were matched to the Spitzer photometry, and the full catalog is available for download through the Spitzer Science Center $^{8}$. For details on the content of the catalog and the nature of the observations, see Gordon et al. (2011).

Throughout, we adopt $A_{\mathrm{V}}=0.12 \mathrm{mag}$ and $E(B-V)=$ $0.04 \mathrm{mag}$ (Schlegel et al. 1998; Harris \& Zaritsky 2004)

\footnotetext{
8 http://data.spitzer.caltech.edu/popular/sage-smc/
}

to account for interstellar reddening. We use the extinction law from Glass (1999) for optical to near-IR bands. Extinction in the Spitzer bands (Indebetouw et al. 2005) is negligible $\left(A_{\lambda}=(6-8) \times 10^{-3} \mathrm{mag}\right.$, for $\lambda=3.6-$ $8 \mu \mathrm{m})$. We assume the distance to the $\mathrm{SMC}$ is $60 \mathrm{kpc}$ (Cioni et al. 2000; Keller \& Wood 2006).

AGB and RSG star candidates were selected photometrically from the catalog, as described by Bover et al. (2011). AGB candidates were separated into carbon-rich (C-AGB), oxygen-rich (O-AGB), extreme (x-AGB), and anomalous O-rich (aO-AGB) sources. The x-AGB sample is dominated by carbon stars (van Loon et al. 1997; van Loon 2006; van Loon et al. 2008; Matsuura et al. 2009), though it likely includes a small number of extreme O-rich sources. The aO-AGB sources are a subclass of the O-AGB candidates with redder $J-[8]$ colors than the bulk O-AGB population at the same magnitude. See Boyer et al. (2011) for a detailed description of their IR properties. Since the nature of the aO-AGB sources is unknown and since Srinivasan et al. (2009) did not distinguish between the aO- and O-AGB sources for the LMC analysis, we lump them together for the massloss analysis (Sect. 2.3).

Some cross-contamination between each stellar type and from young stellar objects (YSOs) and other interlopers is likely, but we expect it to be minimal (see Boyer et al. 2011). Evolved star candidates showing $F_{24} \mu \mathrm{m}>F_{8} \mu \mathrm{m}$ are more likely to include contamination from YSOs or planetary nebulae (Far-IR - FIR - objects in Boyer et al. 2011), so these are considered separately from the AGB and RSG samples.

\subsection{IR excess}

We followed Srinivasan et al. (2009) to estimate the 8- $\mu \mathrm{m}$ excesses $\left(X_{8-\mu \mathrm{m}}\right)$ of the AGB and RSG stars. The $24-\mu \mathrm{m}$ excess was not considered here because $<20 \%$ of the O-rich stars are detected at $24 \mu \mathrm{m}$. See Srinivasan et al. (2009) for a comparison between the 8and $24-\mu \mathrm{m}$ excesses in the LMC. To compute $X_{8-\mu \mathrm{m}}$, we first fit the photospheric emission at optical and near-IR wavelengths to plane-parallel C-rich COMARCS models from Gautschy-Loidl et al. (2004) for the C-AGB stars and the spherical O-rich PHOENIX models from Hauschildt et al. (1999) for the O-AGB, aO-AGB, and RSG stars. We chose one model for each type of star that best fit SEDs with little or no dust. The best-fit model photosphere was then scaled to the $H$-band flux to estimate the IR excess at $8.0 \mu \mathrm{m}$. Luminosities were determined by a simple trapezoidal integration of the $U$ band to $24-\mu \mathrm{m}$ flux.

The $\mathrm{x}-\mathrm{AGB}$ stars and FIR objects are so heavily obscured in the optical as to make it impossible to fit the stellar photosphere. For these sources, we therefore assumed that the flux in the IR is completely dominated by the IR excess. This is a reasonable assumption since we expect $<15 \%$ of the mid-IR flux to come from the stellar photosphere itself in $\mathrm{x}$-AGB stars (for $J-[3.6]>3.7 \mathrm{mag})$.

The dust mass-loss rate, or dust-production rate (DPR), is expected to scale with luminosity as $\tau L$ (e.g., Ivezić \& Elitzur 1995). We therefore expect the DPR $(\dot{D})$ to increase with luminosity (hence evolution), provided optical depth $(\tau)$ does not decrease with evolution 
faster than $L^{-1}$. Figures 7, 8 and 9 from Srinivasan et al. (2009) show that the IR excess also scales with luminosity for AGB stars, making the excess a good proxy for the DPR. The relationship between luminosity and $X_{8-} \mu \mathrm{m}$ in the LMC agrees with what we find in the SMC within the uncertainties.

The $8-\mu \mathrm{m}$ excess may not scale well with the DPR for the dustiest O-rich stars that show silicates in absorption at $10 \mu \mathrm{m}$ rather than in emission. We expect these to be rare in the SMC, where the dust fraction in O-rich stars is low owing to its lower metallicity, and the smaller population results in fewer stars in this short-lived evolutionary phase.

To avoid false detections, Srinivasan et al. (2009) define a threshold for reliable excesses in terms of data quality - only excesses with relative uncertainties less than $1 / 3$ (hereafter, ">3- $\sigma$ excess") were used in the analysis. We follow this convention in the current paper.

\subsection{Derivation of Dust-Production Rates}
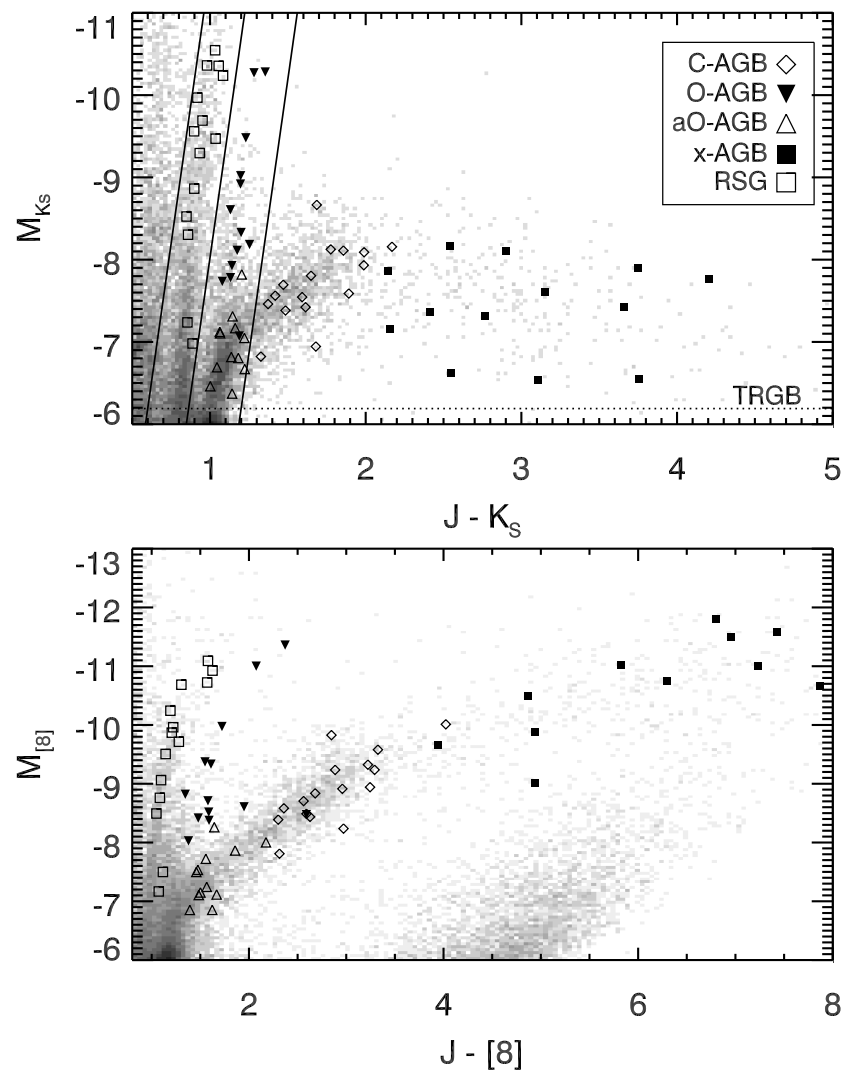

FIG. 1.- Color-magnitude diagrams (CMD) of evolved stars fit with the GRAMS models. The underlying Hess diagram (gray) represents the full SMC catalog. Upper Panel: Near-IR CMD. Solid lines show the approximate division between supergiants, Orich sources, and C-rich sources (cf. Boyer et al. 2011). The dotted line marks the tip of the Red Giant Branch (TRGB). Lower Panel: Mid-IR CMD. Stars were selected for fitting if they have $>3-\sigma$ excess and either an IRS spectrum or $A K A R I$ photometry exists to better constrain the SED. The fitted stars are a representative sample of the SMC evolved stars. Example SEDs are shown in Figure 2

In order to convert the IR excess to the DPR, we require a set of staks with known DPRs in the SMC. Groenewegen et al. (2009b) performed detailed radiative transfer modeling of several stars in the SMC, but these include mainly $\mathrm{x}$-AGB stars and the dustiest O-AGB stars. We therefore select a subset of each type of evolved star and model their SEDs to determine their DPRs. We select sources:

1. that represent SMC AGB and RSG stars over the full IR color space (Fig. 1),

2. that have an $8-\mu \mathrm{m}$ excess with quality $>3 \sigma$,

3. whose photometric classification as O- or C-rich from Bover et al. (2011) does not contradict the spectroscopic classification from van Loon et al. (2008) or Groenewegen et al. (2009b), and

4. that have additional $A K A R I$ photometry and/or Spitzer InfraRed Spectrograph (IRS) spectra to create a better-constrained SED. IRS spectra are from the SMC-Spec Spitzer program (P.I. G. Sloan); see Kemper et al. (2010) for a description of IRS data reduction. AKARI photometry is from Ita et al. (2010).

To find the DPRs of this subset of evolved stars, we use the Grid of RSG and AGB ModelS (GRAMS; Sargent et al. 2011; Srinivasan et al. 2011). These models cover the full range of stellar and dust properties relevant to RSG and AGB stars and were developed to reproduce the IR colors of evolved stars in the LMC. GRAMS fits to the Groenewegen et al. (2009b) carbon stars produce DPRs that are systematically lower by a factor of 2-4 due to a difference in opacities of the amorphous carbon dust used in the two studies (see Fig. 11 in Srinivasan et al. 2011). The GRAMS O-rich fits do not show a systematic offset from the Groenewegen et al. $(2009 \mathrm{~b})$ values; in fact there is an overall agreement between DPRs (Fig. 14 in Sargent et al. 2011). However, it is still possible that fits for individual stars differ in DPRs by a factor of up to 6, especially at lower DPRs (this discrepancy is also due to different optical constants for silicate dust). See Section 2.5 for a discussion of uncertainties.

We fit the SEDs of the selected SMC sources with GRAMS models using a simple chi-squared routine. The chi-squared calculation includes $A K A R I$ photometry, where available. IRS spectra are not included in the chi-squared computation, but are used to confirm by eye whether the best-fit model is a good match. Sources with poor GRAMS fits are excluded from the sample, leaving 12 O-AGB, 16 C-AGB, 13 x-AGB, 12 aO-AGB, and 14 RSG stars. We show a sample of these stars in Figure 2 and their DPRs are listed in Table 1 .

GRAMS assumes a wind expansion velocity of $10 \mathrm{~km} \mathrm{~s}^{-1}$ for all stars. Assuming that $v_{\exp }$ is $10 \mathrm{~km} \mathrm{~s}^{-1}$ for a star with $L=30000 L_{\odot}$ in the LMC, we scaled the DPR from GRAMS according to the following: $\dot{D} \propto$ $L^{0.5} v_{\exp }$ and $v_{\exp } \propto L^{0.25} \psi^{-0.5}$, where $\psi$ is the gas-todust ratio (cf. van Loon et al. 2006). We assume that $\psi$ scales with metallicity (van Loon 2000; Marshall et al. 2004), $Z_{\mathrm{SMC}}=0.2 Z_{\odot}$, and $\psi_{\odot}=200$ (e.g., Knapp et al. 1993; Knapp 2001), so that $\psi_{\mathrm{SMC}}=1000$. However, we note that the gas-to-dust ratio metallicity dependence remains highly uncertain, and may not be the same for Orich and C-rich sources. It has been suggested that C-rich 
TABLE 1

DUST MASS-LOSS RATES FOR STARS FIT WITH GRAMS MODELS

\begin{tabular}{|c|c|c|c|}
\hline Spitzer Designation ${ }^{\mathrm{a}}$ & Name & Type & $\begin{array}{c}\log (\dot{D}) \\
\left(M_{\odot} / \text { yr }\right)\end{array}$ \\
\hline J003656.74-722517.4 & MSX SMC 091 & $\mathrm{x}-\mathrm{AGB}$ & -9.0 \\
\hline J004650.78-714739.2 & MSX SMC 200 & $\mathrm{x}$-AGB & -8.8 \\
\hline J004859.47-733538.7 & IRAS F00471-735 & $\mathrm{x}$-AGB & -8.2 \\
\hline J005000.78-724125.5 & $\ldots$ & $\mathrm{x}-\mathrm{AGB}$ & -9.2 \\
\hline J005036.97-730853.7 & 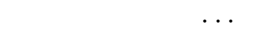 & $\mathrm{x}-\mathrm{AGB}$ & -9.7 \\
\hline J005100.74-722518.5 & MSX SMC 163 & $\mathrm{x}-\mathrm{AGB}$ & -8.3 \\
\hline J005113.57-731036.4 & & $\mathrm{x}-\mathrm{AGB}$ & -8.2 \\
\hline J005446.84-731337.8 & LEGC 105 & $\mathrm{x}$-AGB & -9.2 \\
\hline J005530.99-733752.7 & $\ldots$ & $\mathrm{x}-\mathrm{AGB}$ & -8.9 \\
\hline J005548.54-724729.3 & & $\mathrm{x}$-AGB & -8.8 \\
\hline J005554.63-731136.4 & RAW 960 & $\mathrm{x}-\mathrm{AGB}$ & -9.4 \\
\hline J005718.12-724235.2 & & $\mathrm{x}-\mathrm{AGB}$ & -8.3 \\
\hline J010453.13-720403.9 & 2MASS J010453 & $\mathrm{x}$-AGB & -7.9 \\
\hline J004326.48-732643.3 & 2MASS J004326 & C-AGB & -9.8 \\
\hline J004912.76-732525.5 & $\ldots$ & C-AGB & -10.3 \\
\hline J004934.01-730837.8 & $\cdots$ & C-AGB & -9.8 \\
\hline J004936.46-730357.1 & $\cdots$ & C-AGB & -10.4 \\
\hline J004956.07-724201.3 & $\ldots$ & C-AGB & -10.3 \\
\hline J005015.40-733034.7 & $\ldots$ & C-AGB & -10.1 \\
\hline J005116.41-724256.0 & $\cdots$ & C-AGB & -10.0 \\
\hline J005127.37-724449.3 & & C-AGB & -10.2 \\
\hline J005140.46-725728.9 & MSX SMC 142 & C-AGB & -9.5 \\
\hline J005607.83-731342.6 & & C-AGB & -10.1 \\
\hline J005617.52-722704.3 & $\mathrm{S}^{3} \mathrm{MC} 204803$ & C-AGB & -9.8 \\
\hline J005641.02-724831.0 & $\ldots$ & C-AGB & -9.8 \\
\hline J005753.29-724343.1 & $\ldots$ & C-AGB & -10.4 \\
\hline J005918.41-722734.2 & $\ldots$ & C-AGB & -9.8 \\
\hline J005939.00-722308.0 & $\ldots$ & C-AGB & -10.3 \\
\hline J010029.38-722257.6 & & C-AGB & -10.4 \\
\hline J003201.63-732234.7 & HV 11223 & O-AGB & -10.0 \\
\hline J004249.83-725511.4 & HV 1366 & O-AGB & -10.1 \\
\hline J004843.11-730444.0 & $\ldots$ & O-AGB & -10.7 \\
\hline J004921.37-730327.2 & $\ldots$ & O-AGB & -10.0 \\
\hline J004950.76-724350.0 & $\ldots$ & O-AGB & -11.2 \\
\hline J004952.05-730316.0 & $\cdots$ & O-AGB & -10.8 \\
\hline J005027.20-731021.9 & $\cdots$ & O-AGB & -10.8 \\
\hline J005338.83-733318.9 & $\cdots$ & O-AGB & -9.2 \\
\hline J005443.62-733512.4 & & O-AGB & -10.1 \\
\hline J005850.17-721835.5 & HV 12149 & O-AGB & -9.0 \\
\hline J010302.42-720153.0 & & O-AGB & -8.8 \\
\hline J010426.65-723440.1 & HV 1963 & O-AGB & -9.8 \\
\hline J004924.48-724312.4 & $\cdots$ & aO-AGB & -10.3 \\
\hline J005000.01-730853.1 & $\cdots$ & $\mathrm{aO}-\mathrm{AGB}$ & -10.4 \\
\hline J005005.38-730500.1 & $\cdots$ & aO-AGB & -10.6 \\
\hline J005007.31-724329.9 & $\cdots$ & aO-AGB & -10.4 \\
\hline J005051.81-733036.8 & $\cdots$ & aO-AGB & -10.6 \\
\hline J005103.62-724612.4 & $\ldots$ & $\mathrm{aO}-\mathrm{AGB}$ & -10.4 \\
\hline J005109.94-724525.4 & $\ldots$ & $\mathrm{aO}-\mathrm{AGB}$ & -10.5 \\
\hline J005401.07-733535.6 & $\cdots$ & $\mathrm{aO}-\mathrm{AGB}$ & -10.7 \\
\hline J005454.02-730806.3 & $\cdots$ & $\mathrm{aO}-\mathrm{AGB}$ & -10.4 \\
\hline J005542.97-730552.6 & $\cdots$ & aO-AGB & -10.2 \\
\hline J005855.80-723914.5 & $\cdots$ & aO-AGB & -10.2 \\
\hline J005954.05-722219.6 & $\ldots$ & $\mathrm{aO}-\mathrm{AGB}$ & -10.4 \\
\hline J004846.36-732820.7 & $\ldots$ & RSG & -9.8 \\
\hline J004953.78-730746.2 & $\cdots$ & RSG & -10.9 \\
\hline J005006.34-732811.0 & MSX SMC 096 & RSG & -9.5 \\
\hline J005021.22-730609.5 & . & RSG & -10.8 \\
\hline J005022.38-730755.2 & $\ldots$ & RSG & -11.1 \\
\hline J005047.17-724257.7 & .. & RSG & -10.1 \\
\hline J005049.57-724154.1 & $\ldots$ & RSG & -10.8 \\
\hline J005118.24-724324.7 & $\ldots$ & RSG & -10.8 \\
\hline J005916.83-722511.2 & $\cdots$ & RSG & -11.1 \\
\hline J005934.99-720406.4 & $\cdots$ & RSG & -9.2 \\
\hline J005940.53-722055.9 & $\cdots$ & RSG & -11.1 \\
\hline J010204.06-722610.9 & PMMR 132 & RSG & -10.5 \\
\hline J010304.34-723413.0 & PMMR 141 & RSG & -10.5 \\
\hline $\mathrm{J} 010315.45-724012.2$ & PMMR 145 & RSG & -10.7 \\
\hline
\end{tabular}

a The Spitzer designation prefix is SSTISAGEMA.

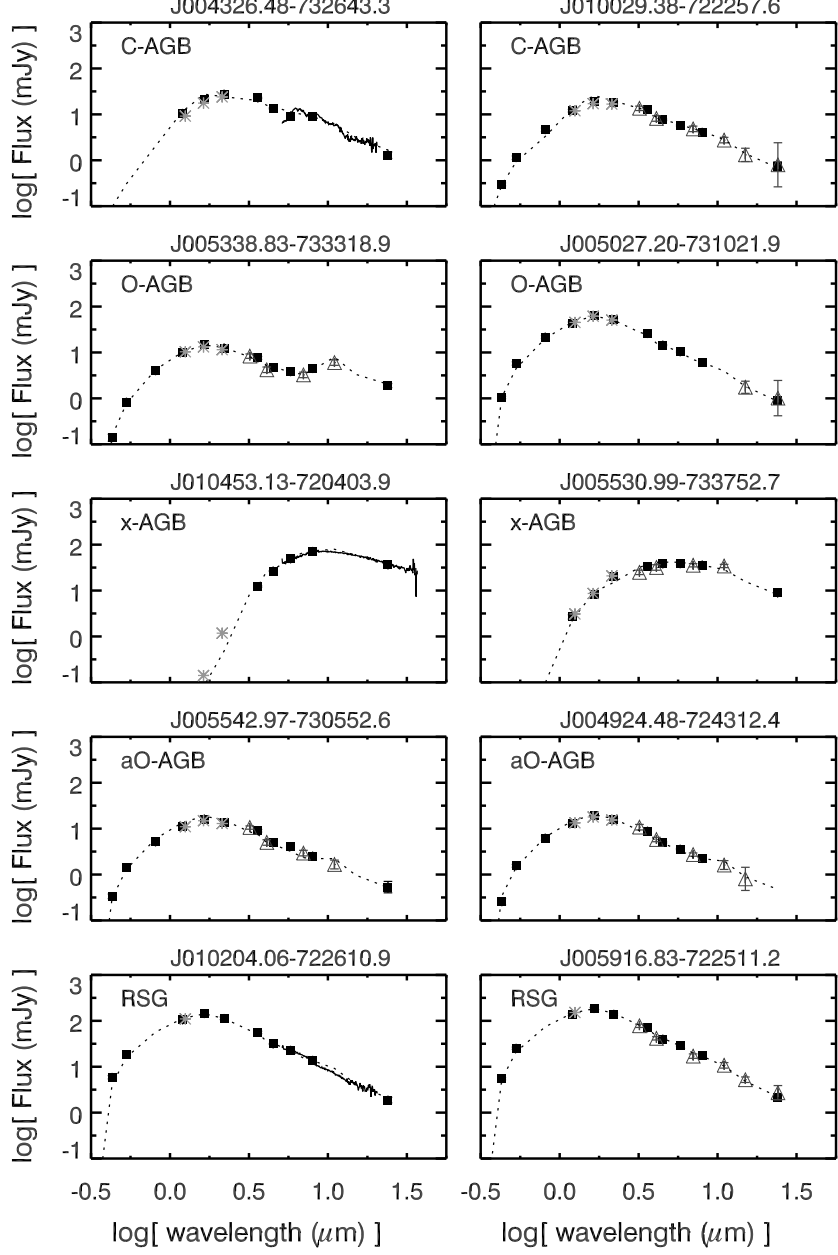

FIG. 2.- Sample SEDs for stars fit with GRAMS models. Photometry from MCPS, 2MASS, and Spitzer is marked by filled squares. Near-IR photometry from the IRSF is marked by asterisks, and $A K A R I$ photometry is marked by open triangles. Spitzer IRS spectra are plotted with solid lines, and the best-fit GRAMS model is marked with dotted lines connecting synthetic photometry at the observed wavelengths.

stars may have gas-to-dust ratios similar to Galactic values, even when in metal-poor environments (e.g., Habing 1996; Groenewegen et al. 2007). For C-rich sources, we therefore assume $\psi=200$.

We note that the GRAMS grid does not account for metallic iron dust, though recent work (McDonald et al. 2010, 2011a, b) shows that it may be an important contributor to dust production, especially for low-mass, metal-poor stars. The inclusion of metallic iron may provide substantial changes to the modeled DPRs for the fainter AGB stars; the direction of this change depends on competing factors relating to the higher opacity of metallic iron dust. Modeling of O-rich stars in the globular cluster $\omega$ Centauri suggests that the inclusion of metallic iron in a purely dust-driven wind will increase the DPRs by approximately a factor of 2 (McDonald et al. 2009, 2011b). However, we expect metallic iron to be only a minor component of optically thick dust shells, which dominate the dust production.

\subsection{Extrapolation to the Entire $S M C$}

In Figure 3, we show the DPRs as a function of $8-\mu \mathrm{m}$ excess for the stars fit in the previous section. The best 
fits in Figure 3 are as follows:

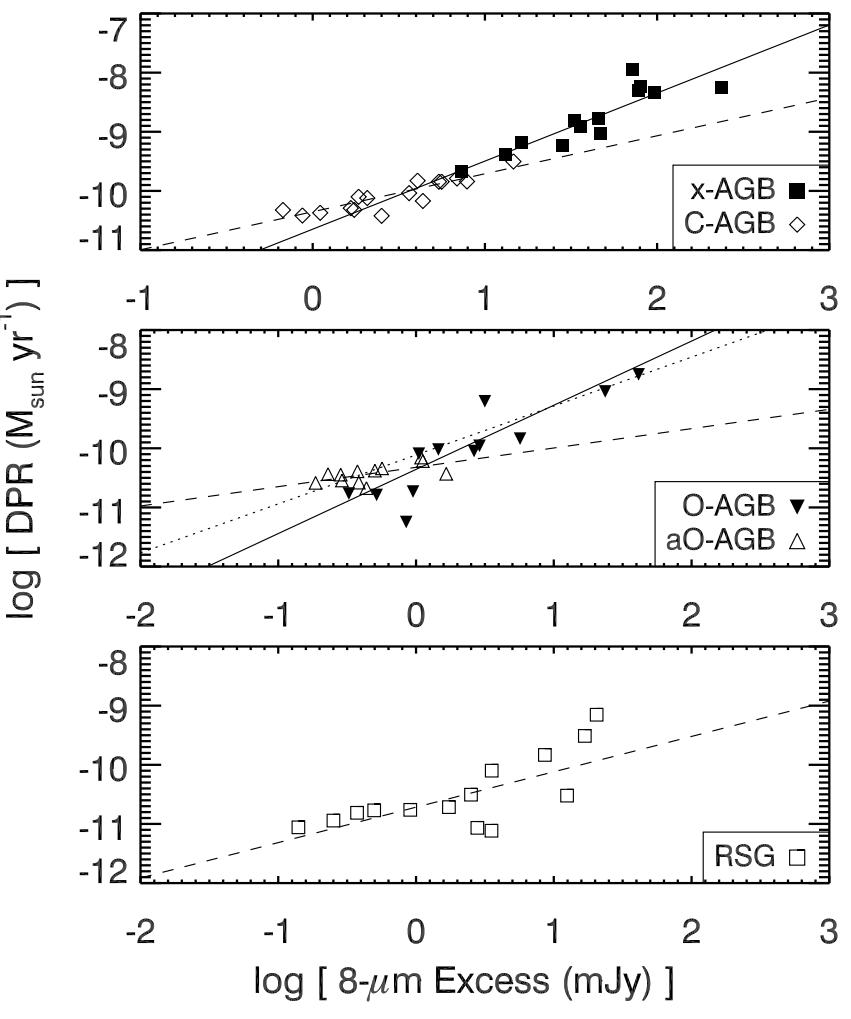

FIG. 3. $-8-\mu \mathrm{m}$ excess vs. $\dot{D}$ for stars marked in Fig. 1 DPRs were determined by applying the GRAMS model grid (see text). The solid lines mark the best fits for the filled symbols, and the dashed lines mark the best fits for the open symbols. In the middle panel, the dotted line marks the best fit obtained by combining the O-AGB and aO-AGB stars. We use the combined fit to derive the DPRs of the entire O-rich AGB sample to facilitate a direct comparison to the LMC analysis, which did not distinguish between aO- and O-AGB sources (Srinivasan et al. 2009).

$$
\log \dot{D}\left(M_{\odot} \mathrm{yr}^{-1}\right)=A+B \log X_{8-\mu \mathrm{m}}(\mathrm{mJy}),
$$

where

$$
\begin{aligned}
& A=-10.7, B=1.2(\mathrm{x}-\mathrm{AGB} / \mathrm{FIR}), \\
& A=-10.4, B=0.6(\mathrm{C}-\mathrm{AGB}), \\
& A=-10.4, B=1.1(\mathrm{O}-\mathrm{AGB} / \mathrm{aO}-\mathrm{AGB}), \\
& A=-10.7, B=0.6(\mathrm{RSG}) .
\end{aligned}
$$

The DPR of each evolved star in the SMC is estimated from its $8-\mu \mathrm{m}$ excess by applying the above $X_{8-\mu \mathrm{m}}-\dot{D}$ relationship. Assuming the FIR objects are dusty evolved stars, their DPRs are derived using the x-AGB $X_{8-\mu \mathrm{m}}-\dot{D}$ fit, as most of the FIR sources fall within the same midIR color-magnitude locus as the x-AGB stars. However, we stress that the majority of the FIR sources are likely YSOs.

Srinivasan et al. (2009) considered only the x-AGB, C$\mathrm{AGB}$, and O-AGB candidates in their analysis of the LMC. Here, we recompute the DPRs for the LMC, separating the aO-AGB and FIR sources and including the RSGs. These DPRs were computed using the $X_{8-\mu \mathrm{m}}-\dot{D}$

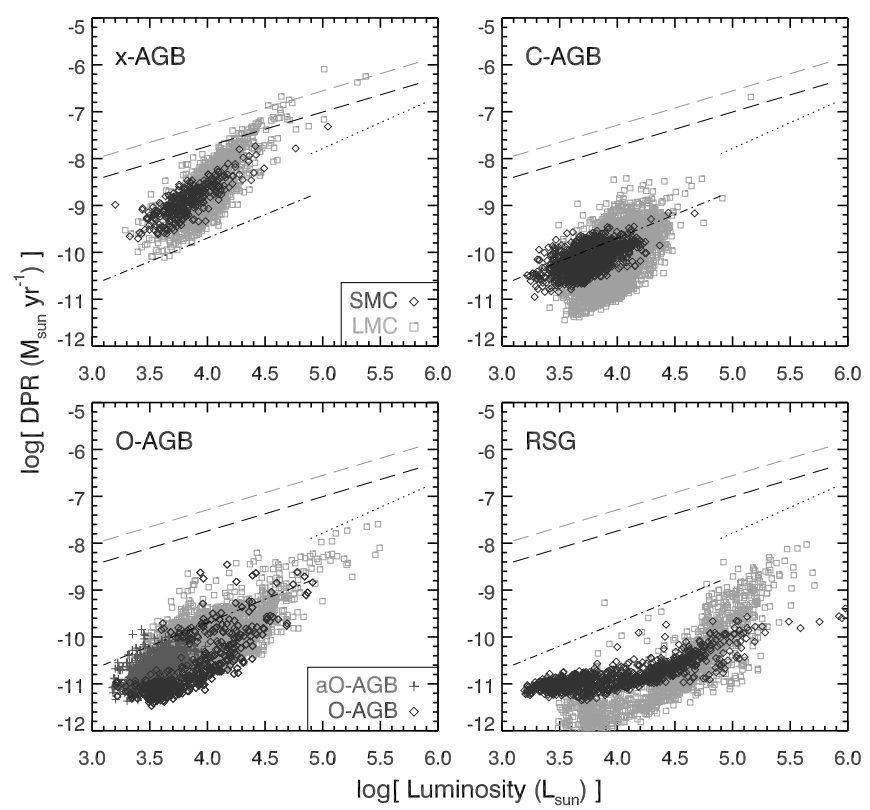

FIG. 4.- The DPR as function of luminosity for the SMC and LMC. The long-dashed line indicates the classical mass loss limit (Jura 1984, $\left.\dot{D}=L\left(v_{\exp } c\right)^{-1} \propto L^{0.75}\right)$ scaled by the metallicity of the LMC (grey boxes) and SMC (black diamonds), the dashdot line indicates the nuclear consumption rate for the CNO cycle, and the dotted line marks the nuclear consumption rate for the $3 a$ process. Both nuclear consumption rates are directly proportional to $L$. LMC and SMC sources are plotted with light and dark points, respectively. Only sources with $>3-\sigma$ excess are included. We note that RSGs with $\log [L / L \odot] \lesssim 4$ are more likely to be AGB stars.

relationship derived by Srinivasan et al. (2009). As with the SMC, the aO-AGB star DPRs are computed using the O-AGB relation, and the FIR object DPRs (assuming they are evolved stars) are computed using the $\mathrm{x}$ AGB relation. The RSGs from van Loon et al. (1999), van Loon et al. (2005), and Groenewegen et al. (2009b) are used to calibrate Equation [1, resulting in:

$$
A=-11.1, B=1.2 \text { (LMC RSGs). }
$$

The resulting DPRs for both galaxies are plotted against luminosity in Figure 4. We see that all of the $\mathrm{x}$-AGB stars lose mass at a rate that is higher than the nuclear consumption rate (dash-dot and dotted lines in Fig. (4), implying that mass loss dominates their subsequent evolution. The same is true for a subset of the C-AGB and O-AGB stars.

\subsection{The Cumulative Dust-Production Rates}

The cumulative DPRs are shown in Figure 5 and the total dust inputs are listed in Tables 2 and 3 . The cumulative DPRs in both galaxies follow similar trends. The $\mathrm{x}$-AGB stars dominate the total DPR at $L>4-$ $8 \times 10^{3} L_{\odot}$. The O-rich stars dominate the dust input at lower luminosities, and the regular C-AGB and the Orich $\mathrm{AGB}$ stars $(\mathrm{O}-\mathrm{AGB}+\mathrm{aO}-\mathrm{AGB})$ contribute similar total DPRs (differing by a factor of 1 in the LMC and 1.6 in the SMC). The FIR objects play a much larger role in the LMC, especially at luminosities higher than the classical AGB limit. This is likely due to increased contamination from YSOs in the LMC, which has a higher star formation rate. 


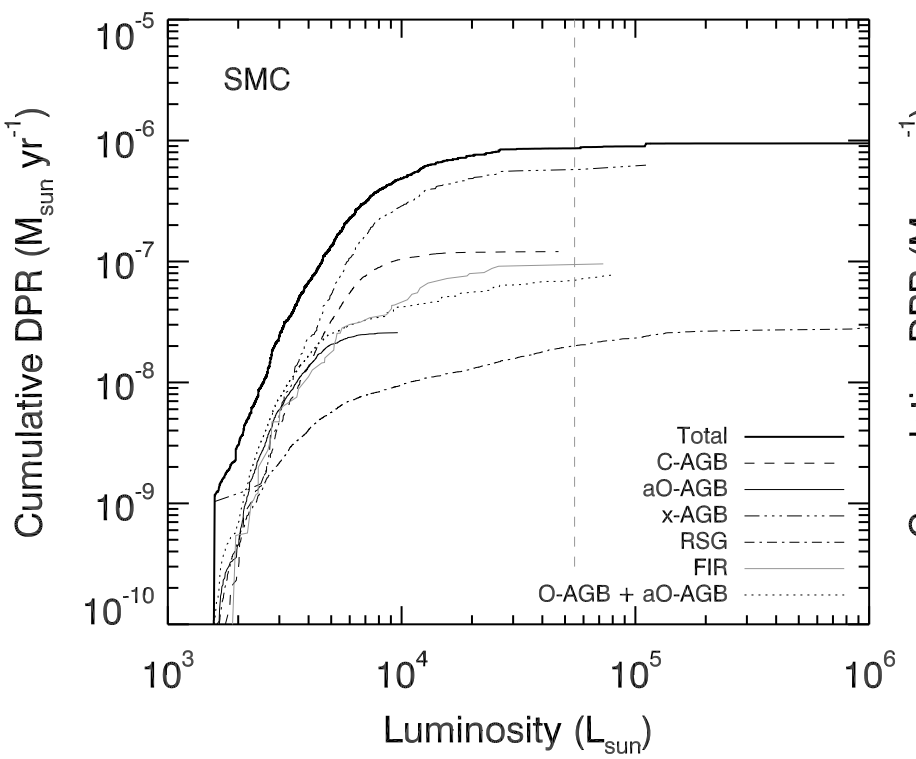

FIG. 5.- Cumulative DPR vs. luminosity for the SMC (left) and LMC (right). The vertical dashed line marks the classical AGB limit.

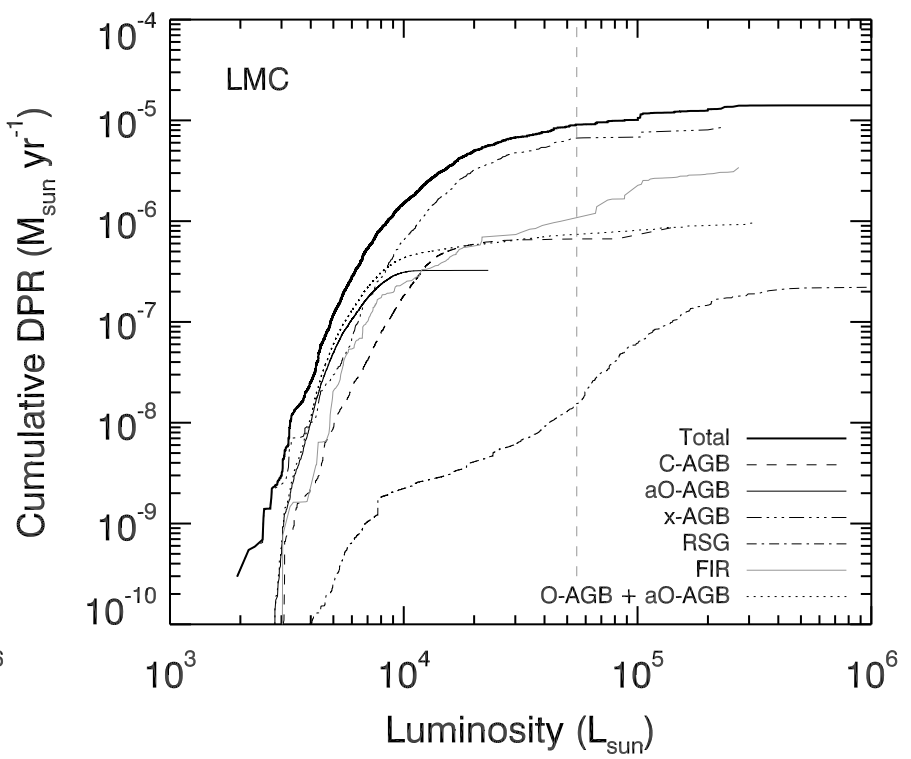

due to an incorrect assumption about the circumstellar dust composition. Groenewegen (2006) shows that if carbon stars have less $\mathrm{SiC}$ and more amorphous carbon, for instance, the color-derived DPRs will decrease, bringing them closer to the excess-derived DPRs. SiC abundance is known to decrease with metallicity (e.g., Lagadec et al. 2007), so is expected to be uncommon in SMC stars. For O-rich stars, a lack of metallic iron or impurities in the silicates will decrease the 3.6- $\mu \mathrm{m}$ flux and a change in the shape of the $10-\mu \mathrm{m}$ silicate feature will affect the $8-\mu \mathrm{m}$ flux; both will affect how the [3.6] - [8] color scales with the DPR. Therefore, with the proper assumptions about dust composition, IR colors may provide DPRs similar to our excess-derived DPRs.

\subsection{Uncertainties in the Dust-Production Rates}

The results and analysis presented in this paper are very sensitive to the DPRs estimated by fitting the observed SEDs to GRAMS models. Some parameters, such as the outflow velocity, are unknown, so it is impossible to determine a formal uncertainty in the DPRs. In this section, we list the known uncertainties associated with the DPR measurements. Some of these were already discussed in Sargent et al. (2011) and Srinivasan et al. (2011), but we repeat them here for completeness.

The current version of the GRAMS grid is computed for spherical dust shell geometry, assuming a constant DPR throughout the AGB lifetime. A treatment of the superwind phase is not considered at present. The DPR is computed from the dust shell inner radius, the sizeand composition-averaged dust grain opacity and the outflow velocity in the shell (cf. Eq. 2 in Srinivasan et al. 2010). When comparing the GRAMS-predicted DPRs with predictions from other studies, it is important to account for the differing choices of dust optical constants; as mentioned in Section 2.2 .

In GRAMS, the outflow velocity is assumed constant and is fixed at $10 \mathrm{~km} \mathrm{~s}^{-1}$, typical of metalpoor stars (Marshall et al. 2004). Here, we scale the DPR to account for the luminosity and the gas-to- 
dust ratio (and hence metallicity) dependence of $v_{\text {exp }}$ (Sec. 2.2, e.g., Habing et al. 1994; Ivezić \& Elitzur 1995; van Loon et al. 2008; Wachter et al. 2008; Lagadec et al. 2010).

In performing SED fitting to individual stars using the GRAMS models, it is necessary to take into account the uncertainties due to photometric errors as well as stellar variability. This results in a range of possible model fits, which can be translated to an uncertainty in the fit parameters, such as luminosity and DPR. A detailed SED-fitting study (D.Riebel et al., in preparation) finds that the relative error on the best-fit DPR is $10 \%-50 \%$ for the entire sample of significant mass-losing sources in the LMC. We expect a similar range of uncertainties in the DPRs used here, though this uncertainty is statistical and diminishes by integrating over an ensemble. Also, the relative uncertainty in comparing the SMC with the LMC is not as large as the absolute uncertainty in each of their DPRs, owing to the similarity of the data (photometry source, distance, and morphological type of the galaxies) and the treatment with the same model grid.

The uncertainties discussed above relate to the determination of DPRs for individual stars in our sample. The $X_{8-\mu \mathrm{m}}-\dot{D}$ relations used in this paper are power-law fits to a representative sample of sources in the SMC (Fig. 3), and there is some uncertainty in these fits. For instance, a slope that is $5 \%$ shallower for the $X_{8-\mu \mathrm{m}}-\dot{D}$ relation (such that B is $5 \%$ smaller in Eq. 1) would result in DPRs $10-30 \%$ lower and result in a higher total contribution from stars with low DPRs.

An additional source of uncertainty lies in our exclusion of binarity and the assumption of a spheroidal envelope. In both cases, the inclination angle could affect the observed $8-\mu \mathrm{m}$ excess. It is impossible to determine binarity and/or geometry from IR photometry alone, but since this analysis includes a large sample of stars (presumably with no preferred inclination angle with respect to the line-of-sight), this is not expected to be a large effect.

\section{DISCUSSION}

\subsection{Circumstellar Dust at Low Metallicity}

In Tables 2 and 3, we show the average DPRs for stars in the LMC and SMC, and in Figure 4, we show how the DPR scales with luminosity. Some of the scatter in Figure 4 is due to a spread in the evolutionary phase at each luminosity. LMC RSGs, which are exclusively O-rich, lose dust at a rate that is up to $10 \times$ higher than $\mathrm{SMC}$ RSGs at the high-luminosity end $\left(\log \left[L / L_{\odot}\right] \gtrsim 4.5\right)$, consistent with the findings from several works showing that O-rich dust is more difficult to form at low metallicity (van Loon 2000, 2006; Sloan et al. 2008). SMC RSGs with low luminosity appear to have higher DPRs than their LMC counterparts. This is likely due to uncertainty in the slope of the $X_{8-\mu \mathrm{m}}-\dot{D}$ relationship (Sec. 2.5).

Previous studies find that the DPR is lower in metalpoor C-rich stars (van Loon 2000; van Loon et al. 2008). On average, our findings agree with this, with mean DPRs 2.2-4.9 times higher in the LMC (Tables 2 and 3). However, Figure 4 shows that the LMC C-rich stars can reach both high and low DPRs compared to the SMC, and it is clear that metal-poor C-rich AGB stars can still be prolific dust producers (e.g., Sloan et al. 2009).
Using a similar treatment of the DPR, Groenewegen et al. (2007) find no clear metallicity dependence among Magellanic Cloud carbon stars. Since our individual DPRs are scaled by the luminosity and gas-to-dust ratio, they are a factor of 3-9 lower than those from Groenewegen et al. (2007), though this should not change the relative DPRs in the SMC and LMC, since we use the solar gas-to-dust ratio $(\psi=200)$ for the carbon stars in both galaxies. These results highlight the importance of AGB dust production at high redshift, where C-rich AGB stars can contribute strongly to the stellar dust production.

\subsection{Gas Input from Cool Evolved Stars}

The total current dust-injection rate from the cool evolved stars in the $\mathrm{SMC}$ is $(8.6-9.5) \times 10^{-7} M_{\odot} \mathrm{yr}^{-1}$, depending on the number of FIR objects which are evolved stars. Assuming that the gas-to-dust ratio $(\psi)$ scales with metallicity for all stars (van Loon 2000; Marshall et al. 2004), then $\psi_{\mathrm{SMC}}=1000$ (see Sec. 2.2). This yields a total gas return of $\left(8.6^{-9.5}\right) \times 10^{-4} M_{\odot} \mathrm{yr}^{-1}$. Using Galactic metallicities for the C-rich objects $(\psi=$ 200) and $\psi=1000$ for the O-rich stars (see Sec. 2.2) yields a total gas return of $(2.6-2.8) \times 10^{-4} M_{\odot} \mathrm{yr}^{-1}$, which is close to the evolved star gas return estimates (derived from dusty evolved stars) in more metal-poor dwarf irregular galaxies with similar mass to the SMC such as WLM and IC $1613\left(\Sigma \dot{D}=(3-7) \times 10^{-4} M_{\odot} \mathrm{yr}^{-1}\right.$, or $\approx 10^{-4} M_{\odot} \mathrm{yr}^{-1}$ if C-rich sources are adjusted to solar metallicity; Boyer et al. 2009b).

\subsection{Other Dust Sources in the SMC}

We expect additional dust input from SNe and hot massive stars such as luminous blue variables (LBVs) and Wolf-Rayet (WR) stars, though the exact amount is highly uncertain. SNe can destroy dust as well as produce it. The SN dust destruction rate is also highly uncertain, and a large fraction of SN dust may not survive long enough to mix with the ISM (see Sec. 3.4). However, several SNe appear to harbor small amounts of dust. This includes $0.02 M_{\odot}$ of dust around SN 2003gd in NGC 628 (Sugerman et al. 2006; Ercolano et al. 2007), 0.02-0.05 $M_{\odot}$ around Cas A (Rho et al. 2008), 2.2-3.4 × 10 ${ }^{-3} M_{\odot}$ around SN 2006jc in UGC 4905 (Sakon et al. 2009), $1 \times 10^{-3} M_{\odot}$ in the Crab SN remnant (Temim et al. 2006), and $3 \times$ $10^{-3} M_{\odot}$ around the SMC SN remnant 1E 0102.2-7219 (Sandstrom et al. 2009). Surprisingly, recent Herschel far-IR and ground-based sub-mm observations reveal a massive reservoir of cold dust around SN 1987A of $0.4-$ $0.7 M_{\odot}$ (Matsuura et al. 2011; Lakićević et al. 2011). Based on these observations and excluding SN 1987A, we might expect SNe to produce $\sim(0.1-5) \times 10^{-2} M_{\odot}$ of dust, on average.

Filipović et al. (1998) estimate the SN rate $\left(\tau_{\mathrm{SN}}\right)$ in the SMC to be about 1 every $350 \pm 70 \mathrm{yr}$. Mathewson et al. (1983) estimate a more conservative rate of 1 SN every 800 yr. These rates combined with the observed range of SN dust mass yields a SN dust input of $(0.1-14) \times$ $10^{-5} M_{\odot} \mathrm{yr}^{-1}$, the lower limit of which is comparable to the input from AGB stars.

The SMC is home to $3 \mathrm{LBVs}$ and 5 supergiant $\mathrm{B}[\mathrm{e}]$ stars that are detected at $24 \mu \mathrm{m}$ with MIPS 
TABLE 4

Dust Sources In The SMC

\begin{tabular}{|c|c|}
\hline Source & $\begin{array}{l}\text { Total Dust Input } \\
\qquad\left(M_{\odot} / \mathrm{yr}\right)\end{array}$ \\
\hline C-rich $\mathrm{AGB}^{\mathrm{a}}$ & $(7.5-8.4) \times 10^{-7}$ \\
\hline O-rich AGB & $7.8 \times 10^{-8}$ \\
\hline RSGs & $3.1 \times 10^{-8}$ \\
\hline $\mathrm{LBV}_{\mathrm{s}}+\mathrm{B}[\mathrm{e}]$ stars $^{\mathrm{b}}$ & $\sim 10^{-6}$ \\
\hline $\mathrm{SNe}^{\mathrm{b}, \mathrm{c}}$ & $(0.13-14.3) \times 10^{-5}$ \\
\hline
\end{tabular}
depends on the fraction of FIR stars which are evolved stars.

$\mathrm{b}$ The dust input from LBVs, B[e] stars, and SNe is highly uncertain. Much of the dust created by massive stars may be ultimately destroyed by the SNe shock. See Section 3.3.

c The range in SNe DPR is computed assuming that SNe input $\sim(0.1-5) \times 10^{-2} M_{\odot}$ of dust, on average, and occur at a rate of 350-800 yr (Sec. 3.3).

(Bonanos et al. 2010), which suggests the presence of circumstellar dust. The dust production by B $[\mathrm{e}]$ stars is not well constrained. Dust inferred from the IR excess may be the remains of a debris disk left over from the formation of the star rather than from dust formation in a stellar outflow. Kastner et al. (2006) estimate the dust mass in the disks around two B[e] stars in the LMC, finding $3 \times 10^{-3} M_{\odot}$ of dust in the disk around R126. If formed by the star, this dust must be ejected in episodic/eruptive events since the mass-loss rate required is much too high to be sustained over the lifetime of a B $[\mathrm{e}]$ star. Each $\mathrm{B}[\mathrm{e}]$ star might thus inject $\sim 10^{-3} M_{\odot}$ over its lifetime. The B $[\mathrm{e}]$ phase lasts $\sim 10^{5} \mathrm{yr}$, amounting to a total dustinjection rate of $10^{-7} M_{\odot} \mathrm{yr}^{-1}$ for $5-10 \mathrm{~B}[\mathrm{e}]$ stars. This is similar to the dust input from C- and O-AGB stars in the SMC.

Boyer et al. (2010) detect a dust-production rate of $2 \times$ $10^{-8} M_{\odot} \mathrm{yr}^{-1}$ around the LBV R71 in the LMC. If this rate is typical of dusty LBVs, then their contribution to the total dust budget is similar to the total DPR of $\mathrm{B}[\mathrm{e}]$ stars. Dust production in LBVs is likely episodic, so if we assume that an LBV might lose a few solar masses of material over its lifetime $\left(\sim 10^{4} \mathrm{yr}\right)$ and assume a gasto-dust ratio of 1000 for the SMC (see Section 3.2), then each LBV might create $0.01 M_{\odot}$ of dust, corresponding to a total DPR of $\sim 10^{-6} M_{\odot} \mathrm{yr}^{-1}$ and matching the input from the cool evolved stars. Galactic LBVs such as AG Carinae and $\eta$ Carinae create 10 times more dust than this due to a smaller gas-to-dust ratio (Voors et al. 2000; Smith et al. 2003).

Table 4 summarizes the estimated dust input from each dust source. We note that much of the dust from massive stars may be destroyed in the ensuing shocks when these stars explode as SNe. Ultimately, the contribution to the total dust budget from massive stars may be negligible. We consider the full range of possibilities in Section 3.4

\subsection{Dust Survival in the ISM}

The dust input into the ISM from stellar sources is listed in Table 4. The dust mass in the SMC ISM inferred from IR and sub-mm imaging is $(0.29-1.1) \times 10^{6} M_{\odot}$ (Bot et al. 2010). The likelihood that the ISM dust is stellar in origin depends on the lifetime of dust in the ISM. Several works find that SN shocks are the dominant dust-destruction mechanism in the ISM (e.g., Draine \& Salpeter 1979; Jones et al. 1994). More than $50 \%$ of a silicate grain $(a=0.1 \mu \mathrm{m})$ is returned to the gas phase in shocks with $v_{\mathrm{s}}>200 \mathrm{~km} \mathrm{~s}^{-1}$ (Draine \& Salpeter 1979). Draine (2009) argue that a typical SN with energy $E_{0}=10^{51} \mathrm{erg}$ in a medium with density $n_{\mathrm{H}}=1 \mathrm{~cm}^{-3}$ will remain in the Sedov-Taylor phase until the shock speed drops to $200 \mathrm{~km} \mathrm{~s}^{-1}$, so we expect a SN to process $M \approx E_{0} / v_{\mathrm{s}}^{2}=1260 M_{\odot}$ of interstellar material. The dust lifetime within the ISM is then:

$$
\tau_{\mathrm{d}}=\frac{M_{\mathrm{ISM}}^{\mathrm{gas}}}{\left(1260 M_{\odot} / \tau_{\mathrm{SN}}\right)},
$$

where $M_{\text {ISM }}^{\text {gas }}\left(\mathrm{H} \mathrm{I}+\mathrm{H}_{2}\right)$ is $4.5 \times 10^{8} M_{\odot}$ (Bolatto et al. 2011). Jones et al. (1996) argue that if the grains are porous, their lifetimes could be enhanced by a factor of 3 . This, combined with Equation 2 and using $\tau_{\mathrm{SN}}$ from Section 3.3. results in an expected dust lifetime of $\tau_{\mathrm{d}}=$ 0.38-0.86 Gyr, similar to the lifetime estimated for the Milky Way ( 0.5 Gyr; Jones et al. 1994, 1996).

It is possible that many $\mathrm{SNe}$ will explode in much denser molecular clouds, with $n_{\mathrm{H}} \sim 10^{3} \mathrm{~cm}^{-3}$. In this case, the Sedov-Taylor phase terminates at higher shock velocities, and the SN may only process $\sim 170 M_{\odot}$ of material, leaving much of the ISM dust unaffected. If all $\mathrm{SNe}$ exploded in dense molecular clouds, then the dust lifetime increases to $\tau_{\mathrm{d}}=2.8-6.7 \mathrm{Gyr}$.

If no stellar dust is destroyed by $\mathrm{SNe}$ (or other mechanisms such as grain-grain collisions), then the lifetime of dust in the ISM is determined by the SFR as $\tau_{\mathrm{d}}=M_{\mathrm{ISM}} / S F R$, where $S F R$ is the star formation rate $\left(3.7 \times 10^{-2} M_{\odot} \mathrm{yr}^{-1} ;\right.$ Bolatto et al. 2011). This yields a lifetime of $\tau_{\mathrm{d}}^{\max }=12 \mathrm{Gyr}$, very near the age of the oldest stellar population in the SMC (Noël et al. 2009). A dust lifetime of 12 Gyr would suggest that most dust that has ever been created in the SMC survives in the ISM today.

In order to compare the dust input to the observed ISM dust mass, we now consider three cases:

(A) massive stars ultimately contribute no dust (it is all destroyed by the SN shock), such that cool evolved stars are the only stellar dust sources,

(B) progenitor dust survives the $\mathrm{SN}$ shock and SNe themselves produce the lower limit of the estimated DPR listed in Table 4, and

(C) progenitor dust survives the $\mathrm{SN}$ shock and SNe themselves produce the upper limit of the estimated DPR listed in Table 4.

Based on the total dust input and the observed ISM dust mass, we can estimate the required dust lifetime for each scenario.

For case A, the total dust input is $(8.6-9.5) \times$ $10^{-7} M_{\odot} \mathrm{yr}^{-1}$ and the dust must therefore survive for $(3-13) \times 10^{11} \mathrm{yr}$, depending on $\tau_{\mathrm{SN}}$, to account for the observed ISM dust mass. This is $2-3$ orders of magnitude longer than the lifetime estimated with Equation 2, and indeed is longer than a Hubble time. Case A is therefore an impossible scenario unless the SMC underwent periods of much higher star formation prior to the era that 
created the current population of AGB stars. In 12 regions spanning the galaxy, Noël et al. (2009) show that the SFR in the SMC remained constant within a factor of 3 from the onset of star formation to intermediate ages, so we do not expect a significantly larger contribution from AGB stars in the past than is currently observed.

For case $B$, the total dust input is $(3.3-3.4) \times$ $10^{-6} M_{\odot} \mathrm{yr}^{-1}$, so the dust lifetime must be $(9-33) \times$ $10^{11} \mathrm{yr}$. This lifetime is also much too long, so the ISM dust cannot be supplied solely by AGB stars and SNe if SNe produce only $10^{-3} M_{\odot}$, on average.

For case $\mathrm{C}$, the total dust input is $1.4 \times 10^{4} M_{\odot} \mathrm{yr}^{-1}$ and the dust lifetime must be 2.1-7.9 Gyr, comparable to the lifetime of porous grains if $\mathrm{SNe}$ shockwaves are the dominant destruction mechanism in the SMC and tend to explode within dense molecular clouds (Eq. 2 $\left.\tau_{\mathrm{d}}=2.8-6.7 \mathrm{Gyr}\right)$. SNe that produce $5 \times 10^{-2} M_{\odot}$ and occur every 350 yr can thus feasibly produce the observed ISM dust mass. We also note that if SNe can produce the amount of dust recently observed around SN 1987A $\left(0.4-0.7 M_{\odot}\right)$, they can explain the ISM dust even for the most pessimistic estimates of the dust lifetime.

\subsection{The SMC Dust Budget: Excess Dust in the ISM?}

It is clear that AGB and RSG stars alone cannot account for the observed ISM dust, even if SNe shocks destroy no dust at all. With certain assumptions, SNe and cool evolved stars together can be made to account for the observed ISM dust mass. This includes assuming that a high fraction of dust created by $\mathrm{SNe}$ and their progenitors survives and that most SNe explode in a dense medium, such that most of the surrounding ISM dust is shielded from destruction. These assumptions are somewhat generous, and we are left with at least some excess of ISM dust, similar to what is seen in the LMC (Matsuura et al. 2009), the Milky Way (Dwek 1998), and in high-redshift galaxies. Assuming the dust lifetime derived from Equation 2, the AGB and RSG stars combined can account for only up to $2.1 \%$ of the ISM dust.

Case B in Section 3.4 assumes a conservative estimate for the SNe dust production. This scenario implies that dust must grow in the ISM itself, as discussed by Draine (2009) and references therein, unless evolved stars produce more dust than is implied by the mid-IR observations. Far-IR imaging of a central $2^{\circ} \times 8^{\circ}$ strip of the LMC with Herschel revealed a strong far-IR excess $(>200 \mu \mathrm{m})$ around only one star, the LBV R71 (Boyer et al. 2010), suggesting that cold dust envelopes are rare around evolved stars. Cool evolved stars such as AGB and RSG stars are thus unlikely to be the solution to the missing dust problem. However, if SN 1987A is an anomaly or destroys its own dust, the dust input from cool evolved stars, especially extreme carbon-rich AGB stars, rivals that from SNe.

Dust accretion in molecular clouds might contribute significantly to the total dust budget (e.g., Dwek 1998; Zhukovska et al. 2008). A chemical evolution model of the Milky Way derived by Zhukovska et al. (2008) finds that the AGB stars dominate the dust input until the metallicity surpasses $Z \approx 10^{-3}$. The SMC metallicity is $Z=(2 \pm 0.7) \times 10^{-3}$ (Luck et al. 1998), so we might expect the dust input from AGB stars to rival the rate of dust growth in molecular clouds. Assuming case B in Section 3.4, we find that dust grains must grow at a rate of $(0.1-3) \times 10^{-3} M_{\odot} \mathrm{yr}^{-1}$, using conservative estimates for the dust lifetime $(0.4-2.2 \mathrm{Gyr})$. This is similar to the rate of dust growth in the Milky Way ISM suggested by Jenniskens et al. (1993) $\left(10^{-3} M_{\odot} \mathrm{yr}^{-1}\right)$. The rate of dust accretion in the ISM may thus be $2-3$ orders of magnitude larger than the AGB dust production.

Alternatively, we must also consider that the ISM recycling timescale may be long enough such that the current stellar dust input is not representative of the stellar input during the epoch that created today's ISM dust. During an increase in star formation, the SNe and RSGs would contribute more to the dust input. Noël et al. (2009) find that the SFR was enhanced 0.2-0.5 Gyr ago in the eastern fields, perhaps corresponding to a close encounter with the LMC, and resulting in a larger rate of SNe dust injection during that era.

It is also possible that the ISM dust mass is overestimated. However, the dust mass assumed here $((0.29$ 1.1) $\times 10^{6} M_{\odot} ;$ Bot et al. 2010) includes the sub-mm excess, which is well-constrained by invoking spinning dust grains. If, instead, the sub-mm excess is due to very cold dust grains, the ISM dust mass would increase dramatically, exasperating the problem.

\section{SUMMARY OF CONCLUSIONS}

We estimate the dust-production rates of cool evolved stars in the SMC using the 8- $\mu \mathrm{m}$ excess and compare the dust input to that from $\mathrm{SNe}$ and to the dust mass in the ISM. We find that the C-rich AGB candidates account for $87 \%-89 \%$ of the total cool evolved star dust input. The equivalent fraction in the LMC is $89 \%-91 \%$. The majority of this dust input comes from the extreme AGB stars. RSG stars play a minor role in the dust input, especially below the classical AGB luminosity limit.

While we can now quantify the dust production in the winds of cool evolved stars, the SNe dust-production rate (and dust-destruction rate) remains poorly constrained. It is possible that SNe can account for all of the ISM dust if they can each produce the upper range of dust masses observed around SN remnants. If, on the other hand, SNe can produce only the smaller dust masses inferred from mid-IR observations of several SN remnants, we expect SNe to contribute equally to the ISM dust compared to the cool evolved stars. This is similar to the findings in the LMC, suggesting only a small variance with metallicity. In this case, an additional dust source is required and perhaps implies that dust grows efficiently in the ISM.

This work is supported by NASA via JPL contracts 130827 and 1340964 .

\section{REFERENCES}

Andrews, J. E., et al. 2011, ApJ, 731, 47

Bolatto, A. D., et al. 2011, ApJ, 741, 12

Bonanos, A. Z., et al. 2010, AJ, 140, 416
Bot, C., et al. 2010, A\&A, 523, 20

Boyer, M. L., et al. 2009a, ApJ, 705, 746

-. 2009b, ApJ, 697, 1993 
-. 2011, AJ, 142, 103

—. 2010, ApJ, 711, L99

Cioni, M., et al. 2000, A\&A, 359, 601

Draine, B. T. 2009, in Astronomical Society of the Pacific

Conference Series, Vol. 414, Cosmic Dust - Near and Far, ed. T. Henning, E. Grün, \& J. Steinacker, 453

Draine, B. T. \& Salpeter, E. E. 1979, ApJ, 231, 438

Dwek, E. 1998, ApJ, 501, 643

Dwek, E. \& Cherchneff, I. 2011, ApJ, 727, 63

Ercolano, B., Barlow, M. J., \& Sugerman, B. E. K. 2007, MNRAS, 375, 753

Filipović, M. D., et al. 1998, A\&AS, 127, 119

Gautschy-Loidl, R., et al. 2004, A\&A, 422, 289

Gehrz, R. 1989, in IAU Symposium, Vol. 135, Interstellar Dust, ed. L. J. Allamandola \& A. G. G. M. Tielens, 445

Glass, I. S. 1999, Handbook of Infrared Astronomy, ed. Glass, I. S.

Gordon, K. D., et al. 2011, AJ, 142, 102

Groenewegen, M. A. T. 2006, A\&A, 448, 181

Groenewegen, M. A. T., Lançon, A., \& Marescaux, M. 2009a, A\&A, 504, 1031

Groenewegen, M. A. T., et al. 2009b, A\&A, 506, 1277

-. 2007, MNRAS, 376, 313

Habing, H. J. 1996, A\&A Rev., 7, 97

Habing, H. J., Tignon, J., \& Tielens, A. G. G. M. 1994, A\&A, 286,523

Harris, J. \& Zaritsky, D. 2004, AJ, 127, 1531

Hauschildt, P. H., Allard, F., \& Baron, E. 1999, ApJ, 512, 377

Indebetouw, R., et al. 2005, ApJ, 619, 931

Ita, Y., et al. 2010, PASJ, 62, 273

Ivezić, Ž. \& Elitzur, M. 1995, ApJ, 445, 415

Jenniskens, P., et al. 1993, A\&A, 273, 583

Jones, A. P., Tielens, A. G. G. M., \& Hollenbach, D. J. 1996, ApJ, 469, 740

Jones, A. P., et al. 1994, ApJ, 433, 797

Jura, M. 1984, ApJ, 282, 200

Kastner, J. H., et al. 2006, ApJ, 638, L29

Kato, D., et al. 2007, PASJ, 59, 615

Keller, S. C. \& Wood, P. R. 2006, ApJ, 642, 834

Kemper, F., et al. 2010, PASP, 122, 683

Knapp, G. R. 2001, in Astronomical Society of the Pacific Conference Series, Vol. 231, Tetons 4: Galactic Structure, Stars and the Interstellar Medium, ed. C. E. Woodward, M. D. Bicay, \& J. M. Shull, 127

Knapp, G. R., Sandell, G., \& Robson, E. I. 1993, ApJS, 88, 173

Lagadec, E., et al. 2010, MNRAS, 403, 1331

—. 2007, MNRAS, 376, 1270
—. 2009, MNRAS, 396, 598

Lakićević, M., et al. 2011, A\&A, 532, L8

Luck, R. E., et al. 1998, AJ, 115, 605

Marshall, J. R., et al. 2004, MNRAS, 355, 1348

Mathewson, D. S., et al. 1983, ApJS, 51, 345

Matsuura, M., et al. 2009, MNRAS, 396, 918

—. 2011, Science, 333, 1258

-. 2007, MNRAS, 382, 1889

McDonald, I., et al. 2011a, ApJ, 730, 71

—. 2010, ApJ, 717, L92

—. 2011b, MNRAS, 417, 20

-. 2009, MNRAS, 394, 831

Noël, N. E. D., et al. 2009, ApJ, 705, 1260

Rho, J., et al. 2008, ApJ, 673, 271

Russell, S. C. \& Dopita, M. A. 1992, ApJ, 384, 508

Sakon, I., et al. 2009, ApJ, 692, 546

Sandstrom, K. M., et al. 2009, ApJ, 696, 2138

Sargent, B. A., Srinivasan, S., \& Meixner, M. 2011, ApJ, 728, 93

Schlegel, D. J., Finkbeiner, D. P., \& Davis, M. 1998, ApJ, 500, 525

Skrutskie, M. F., et al. 2006, AJ, 131, 1163

Sloan, G. C., et al. 2008, ApJ, 686, 1056

- 2009, Science, 323, 353

Smith, N., et al. 2003, AJ, 125, 1458

Srinivasan, S., et al. 2009, AJ, 137, 4810

-. 2010, A\&A, 524, A49

Srinivasan, S., Sargent, B. A., \& Meixner, M. 2011, A\&A, 532, A54

Sugerman, B. E. K., et al. 2006, Science, 313, 196

Temim, T., et al. 2006, AJ, 132, 1610

Valiante, R., et al. 2009, MNRAS, 397, 1661

van Loon, J. Th. 2000, A\&A, 354, 125

van Loon, J. Th. 2006, in Astronomical Society of the Pacific

Conference Series, Vol. 353, Stellar Evolution at Low

Metallicity: Mass Loss, Explosions, Cosmology, ed.

H. J. G. L. M. Lamers, N. Langer, T. Nugis, \& K. Annuk, 211 van Loon, J. Th., et al. 2005, A\&A, 438, 273

-. 2008, A\&A, 487, 1055

-. 1999, A\&A, 351, 559

-. 2006, A\&A, 450, 339

-. 1997, A\&A, 325, 585

Voors, R. H. M., et al. 2000, A\&A, 356, 501

Wachter, A., et al. 2008, A\&A, 486, 497

Zaritsky, D., et al. 2002, AJ, 123, 855

Zhukovska, S., Gail, H.-P., \& Trieloff, M. 2008, A\&A, 479, 453 